\title{
The potential role of VEGF-induced vascularisation in the bony repair of injured growth plate cartilage
}

\section{Rosa Chung, Bruce K Foster ${ }^{1}$ and Cory J Xian}

School of Pharmacy and Medical Sciences, Sansom Institute for Health Research, University of South Australia, City East Campus, GPO Box 2471, Adelaide, South Australia 5001, Australia

'Department of Orthopaedic Surgery, Women's and Children's Hospital, North Adelaide, South Australia 5006, Australia
Correspondence should be addressed to C J Xian

Email

cory.xian@unisa.edu.au

\begin{abstract}
Growth plate injuries often result in undesirable bony repair causing bone growth defects, for which the underlying mechanisms are unclear. Whilst the key importance of pro-angiogenic vascular endothelial growth factor (VEGF) is well-known in bone development and fracture repair, its role during growth plate bony repair remains unexplored. Using a rat tibial growth plate injury repair model with anti-VEGF antibody, Bevacizumab, as a single i.p. injection $(2.5 \mathrm{mg} / \mathrm{kg})$ after injury, this study examined the roles of VEGF-driven angiogenesis during growth plate bony repair. Histology analyses observed isolectin- $B_{4}$-positive endothelial cells and blood vessel-like structures within the injury site on days 6 and 14, with anti-VEGF treatment significantly decreasing blood-vessel-like structures within the injury site $(P<0.05)$. Compared with untreated controls, anti-VEGF treatment resulted in an increase in undifferentiated mesenchymal repair tissue, but decreased bony tissue at the injury site at day $14(P<0.01)$. Consistently, microcomputed tomography analysis of the injury site showed significantly decreased bony repair tissue after treatment $(P<0.01)$. RT-PCR analyses revealed a significant decrease in osteocalcin $(P<0.01)$ and a decreasing trend in $R u n \times 2$ expression at the injury site following treatment. Furthermore, growth plate injury-induced reduced tibial lengthening was more pronounced in anti-VEGF-treated injured rats on day 60 , consistent with the observation of a significantly increased height of the hypertrophic zone adjacent to the growth plate injury site $(P<0.05)$. These results indicate that VEGF is important for angiogenesis and formation of bony repair tissue at the growth plate injury site as well as for endochondral bone lengthening function of the uninjured growth plate.
\end{abstract}

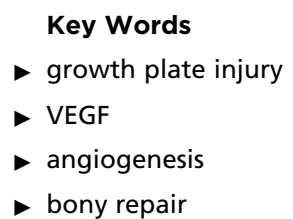

Journal of Endocrinology (2014) 221, 63-75

\section{Introduction}

The growth plate cartilage is responsible for achieving the longitudinal growth of long bones. Being a cartilage scaffold, the growth plate remains the weakest part of a long bone and hence prone to injury (Mizuta et al. 1987). Following an injury, the growth plate is often repaired by an 'unwanted' bony tissue (also called a bone bridge) causing orthopaedic problems such as limb length discrepancy and bone angulation deformity (Salter \& Harris 1963, Ogden $2000 a, b, c$, Xian et al. 2004, Zhou et al. 2004, Chung et al. 2006, 2011, McCarty et al. 2010, Fischerauer et al. 2011, Macsai et al. 2011). Although a microarray gene expression study (Macsai et al. 2012) and 
pathway-specific studies were recently reported describing changes in the levels of gene expression and potential roles of Wnt/ $\beta$-catenin (Chung et al. 2013a,b) and protein kinase-D (Chung et al. 2013a,b) at different stages of bony repair in a rat model, the underlying pathobiology for the undesirable bony repair is unclear, and the resulting bone growth defects currently lack biological preventative treatments (Xian \& Foster 2006, Chung et al. 2011). Improvement of our understanding of the underlying mechanisms leading to the undesirable bony repair is required, which may aid in the development of a future preventative biological treatment (Xian \& Foster 2006, Chung et al. 2011).

Results from previous studies have indicated that the bone bridge formation within the growth plate injury site occurs via direct (intramembranous ossification) and indirect (endochondral ossification) bone formation mechanisms (Xian et al. 2004, Arasapam et al. 2006, Chung et al. 2006, Zhou et al. 2006) as well as the process of angiogenesis within the injury site which is crucial for both types of bone formation (Hausman et al. 2001). It is known that, after a 'fibrous tissue' is formed from the infiltrated stromal cells at an injured growth plate, its invasion by new blood vessels (angiogenesis) is a prerequisite for its osseous transformation (Ogden 2000a,b,c). Angiogenesis is important for providing oxygen, vital nutrients and cells essential for successful bone development, growth as well as fracture repair (Carano \& Filvaroff 2003, Keramaris et al. 2008, Tang et al. 2012). Although fibroblast growth factor 2, platelet-derived growth factor (PDGFBB) and vascular endothelial growth factor (VEGF) have all been associated as angiogenic growth factors, VEGF has been identified as the key mediator for angiogenesis (Ogden 2000a,b,c, Keramaris et al. 2008, Beamer etal. 2010). In addition to VEGF's roles in the migration and proliferation of endothelial cells and blood vessel formation (Carlevaro et al. 2000), VEGF receptors have also been localised on osteoblasts (Midy \& Plouet 1994, Deckers et al. 2000) and osteoclasts (Nakagawa et al. 2000, Street et al. 2002) - indicating the potential importance of VEGF in both angiogenesis as well as osteogenesis and bone remodelling. Suggestive of VEGF's role in bone fracture healing, spatial and temporal expression of VEGF was observed throughout bone fracture repair (Ferguson et al. 1999), and the addition of exogenous VEGF accelerated bone regeneration in vivo, and encouraged formation of bone-forming cells (osteoblastogenesis) in vitro (Midy \& Plouet 1994, Street et al. 2002, Geiger et al. 2005). Furthermore, over-expression of VEGF in bone resulted in an increase in the amount of bone formation
(Zelzer et al. 2004). Conversely, the inactivation of VEGF in mice resulted in decreased trabecular formation during bone growth (Gerber et al. 1999, Haigh et al. 2000) and impaired bone fracture repair (Street et al. 2002). Thus, it is clear that VEGF is critical for angiogenesis, bone growth and bone fracture repair.

While a recent study has observed the expression of VEGF and its receptors during growth plate injury repair with a similar spatial and temporal pattern to that in bone fracture repair (Fischerauer et al. 2011), the role of VEGF in the angiogenesis and the bony repair of the injured growth plate remains unknown. In the current study, it is proposed that VEGF is critical for the angiogenesis and bony repair within the growth plate injury site, and that blocking of VEGF will decrease the undesirable bony repair tissue following growth plate injury. To address this, the current study examined the effects of blocking VEGF activity using a clinically available anti-VEGF humanised antibody, Bevacizumab, in a rat tibia growth plate injury repair model. Bevacizumab binds directly to VEGF protein, blocking VEGF binding to its receptors and thus inhibiting angiogenesis. Bevacizumab has been found to have a half-life of 1-2 weeks in rats (Lin et al. 1999), making it an ideal inhibitor for VEGF action for the time frame and focus of this study.

\section{Materials and methods}

\section{Growth plate injury and treatment trial}

Six-week-old male Sprague-Dawley rats were subjected to experimental growth plate injury in the proximal tibia of both hind legs. Briefly, injury was inflicted via a drill-hole method, whereby disruption of the central part of the proximal tibial growth plate cartilage was performed as described by Xian et al. (2004). These rats were stratified and randomly placed in different groups ( $n=8$ per group), receiving, straight after growth plate injury, a single i.p. injection of anti-VEGF antibody (Bevacizumab) (Genentech/Roche, San Francisco, CA, USA) at $2.5 \mathrm{mg} / \mathrm{kg}$ (Ignjatovic et al. 2010), or saline vehicle control. A group of non-injured rats $(n=8)$ which did not receive any treatments were killed on day 14 and were used as normal controls. Furthermore, two groups of non-injured rats $(n=6)$ receiving vehicle only or a single injection of antiVEGF were killed on day 60 and were used as day 60 normal controls or anti-VEGF-treated non-injured controls respectively. The protocol was carried out under the guidelines of the National Health and Medical Research Council of Australia and with approval from

Published by Bioscientifica Lto. 
the Animal Ethics Committee of the SA Pathology (IMVS), South Australia.

Rats were killed (by $\mathrm{CO}_{2}$ overdose) for specimen collection at days 6,14 and 60 post surgery. For day 6 and 14 groups, both tibiae were dissected and cleared of soft tissues. For all day 60 rats ( $n=6$ rats/group), tibial length measurements were made at surgery and at killing for examination of the overall long-term effects of both injury and anti-VEGF treatment. The left proximal tibia was snapped-opened gently at the metaphyseal/growth plate border, exposing the growth plate cartilage and the growth plate injury site. The exposed injury site (which is not integrated well with the adjacent uninjured cartilage) was collected carefully using a small sterile surgical blade. Immediately after collection, the samples were snap-frozen and stored at $-80^{\circ} \mathrm{C}$ until use for RNA extraction. The right proximal tibia was fixed in 10\% formalin for $24 \mathrm{~h}$ at $4{ }^{\circ} \mathrm{C}$ and then wrapped in saline-soaked gauze and frozen at $-80^{\circ} \mathrm{C}$ until use for microcomputed tomography (microCT) analysis (see below). Following microCT scans, bones were immediately decalcified for 14 days in Immunocal solution (Decal Corporation, New York, NY, USA), bisected longitudinally through the growth plate injury site, and processed for paraffin-embedding. Sections of $4 \mu \mathrm{m}$ thick were cut from paraffin tissue blocks and mounted on SuperFrost Plus glass slides for further histological immunostaining as well.

\section{ELISA for Bevacizumab}

To monitor anti-VEGF levels in rats treated with anti-VEGF, ELISA was carried out with serum of blood samples collected on days of killing (days 6 and 14). Briefly, microplate wells were coated with $100 \mu \mathrm{l}$ of human VEGF $_{165}$ peptide (Cell Signalling Technology, Beverley, MA, USA) at a concentration of $0.15 \mathrm{mg} / \mathrm{l}$ overnight at $4{ }^{\circ} \mathrm{C}$. Following washes and then blocking for $2 \mathrm{~h}$ at room temperature, the plate was then washed and $100 \mu$ l of serum samples or Bevacizumab (human imunoglobulin G (IgG) against VEGF) standards of various dilutions was added. Following incubation for $2 \mathrm{~h}$ and then washes, $100 \mu \mathrm{l}$ of horseradish peroxidase (HRP) conjugated goat anti-human IgG (Invitrogen; 1:16000 in blocking buffer) was added. Following incubation for an hour and washes, 3,3',5,5'tetramethylbenzidine was added and incubated for $30 \mathrm{~min}$ for colour reaction before reaction was terminated by adding $100 \mu \mathrm{l}$ of $1 \mathrm{M}$ sulphuric acid. Spectrophotometric absorbance readings were obtained out at 490 and $520 \mathrm{~nm}$. All measurements were performed in duplicate, and negative and positive controls were included for internal validity.

\section{Isolectin- $B_{4}$ staining of blood vessels within the growth plate injury site and the adjacent metaphyseal region}

To examine the effect of anti-VEGF treatment on angiogenesis during growth plate injury repair and on blood vessels in the adjacent metaphyseal bone region, staining of endothelial cell marker, isolectin- $\mathrm{B}_{4}\left(\mathrm{I}-\mathrm{B}_{4}\right)$, was carried out. Briefly, sections were deparaffinised and rehydrated and the endogenous peroxidase in sections was quenched with $0.3 \%$ hydrogen peroxide in methanol for $30 \mathrm{~min}$. Sections were blocked with $1 \%$ BSA in PBS for $10 \mathrm{~min}$ before incubation with biotinylated Griffonia (Bandeiraea) simplicifolia lectin I ( $\left(\mathrm{B}_{4}\right)$ for 30 min (1:100; Vector Labs, Burlingame, CA, USA). After washes, sections were then incubated with HRP-streptavidin (1:700) for $30 \mathrm{~min}$, followed by washes and colour development using liquid DAB solution (Dako, Melbourne, VIC, Australia). The numbers of I- $\mathrm{B}_{4}$-positive vessel-like structures within the growth plate injury were quantified and expressed as average numbers per $\mathrm{mm}^{2}$.

\section{Haematoxylin and eosin, Alcian blue staining and image analysis of tissue repair and adjacent growth plate heights}

In order to visualise the mesenchymal (Me) infiltrate and cartilage or bone tissue formed within the injury site, deparaffinised tissue sections were stained with Alcian blue, haematoxylin and eosin (H\&E). The proportions of each of the different types of repair tissues (Me infiltrate, cartilaginous (Ca) tissue, bone trabeculae (BT) and bone marrow) within the injury site were analysed using an image analysis program Image-Pro Plus (Media Cybernetics, Rockville, MD, USA) (Chung et al. 2009). These tissue types were distinguished on the basis of their morphologies and colours after histological staining as described previously (Xian et al. 2004). The measurements on four separate sections for each sample were then averaged and expressed as percentages of the total injury site area (Chung et al. 2006). In addition, to examine treatment effects on the uninjured areas of growth plate, images of the growth plate areas adjacent to the injury site were taken, and zonal height measurements were done on the resting zone, proliferative zone and hypertrophic zone by image analysis as described previously (Fan et al. 2009).

\section{MicroCT analysis of the growth plate injury site and drill injury tract}

MicroCT was used to assess the bony tissue formation within the growth plate injury site and drill tract.

Published by Bioscientifica Ltd. 
Briefly, scans were run on an average of 3 at a resolution of $9 \mu \mathrm{m}$. Reconstruction and realignment of images for analysis were done using NRecon (v.1.4.4 SkyScan, Kartuizersweg, Kontich, Belgium) and DataViewer (v.1.4.1.0 SkyScan) respectively. Analysis was performed using CTan Software (v.1.9.1.0 SkyScan). For measuring the bone volume within the growth plate injury site, the growth plate injury site was traced for each slice as the 'region of interest' (ROI) followed by the measurements of the amount of bone within the ROI. Bone volume percentage (BV/TV\%) was calculated from 30 scan slices as described previously (Macsai et al. 2011). Threedimensional images were produced using Cvol Software (v.1.9.1.0 SkyScan). Similarly, to examine the effects of the systemically delivered anti-VEGF or vehicle on bone healing at the drill injury track, the BV/TV\% was also calculated in transaxial scan slices (around 280 slices) along the drill tract - from cortical window up to the start of the growth plate. Measurements and calculations were done as described previously on $\sim 280$ scan slices.

\section{Real-time qualitative RT-PCR expression analysis of cartilage- and bone-related genes}

To examine the effect of anti-VEGF treatment on the expression of osteogenesis- and chondrogenesis-related genes, total RNA from growth plate injury site samples was extracted using an Aurum Total-RNA mini-kit (Bio-Rad). Briefly, growth plate injury site samples were lysed in the supplied buffer and passed through needles (21 and 19 gauge). Lysed samples were then run through a column (with on-column DNase treatment) with RNA collected at the end of the process. Total RNA quality and quantity were then determined by using a NanoDrop 2000 spectrophotometer (Thermo Scientific, Scoresby, VIC, Australia). cDNA was then synthesised from the RNA using an iScript cDNA synthesis kit (Bio-Rad). Relative real-time PCR was carried out using gene-specific primer pairs for Runx2 (forward, 5'-TCACAAATCTCCCCAAGTGG-3' and reverse, $5^{\prime}$-GAATGCGCCCTAAATCACTGA-3'), Osteocalcin (forward, 5'-AAGCCTTCATGTCCAAGCAGG-3' and reverse, 5'-AGGCGGTGTTGAAGCCATACT-3'), Sox9 (forward, $5^{\prime}$-AGTACCCGCATCTGCACAAC- $3^{\prime}$ and reverse, $5^{\prime}$-ACTTGTAATCGGGGTGGTCT-3'), type-2 collagen (Col2A; forward, $5^{\prime}$-CTCATCCAGGGCTCCAATGAT- $3^{\prime}$ and reverse, $5^{\prime}$-TCTGTGATCGGTACTCGATGA-3') and Cyclophilin-A (CycA; forward, $5^{\prime}$-GAGCTGTTTGCAGACAAAGTTC- ${ }^{\prime}$ and reverse, 5'-CCCTGGCACATGAATCCTGG-3'). CyclophilinA was used as an internal control (Zhou et al. 2004). Gene expression was measured relative to that of Cyclophilin-A.

\section{Immunohistochemical analysis of Runx2}

Immunohistochemical analysis was done to examine treatment effects on immunolocalisation of the early osteogenic transcription factor, RUNX2, within the injury site as described previously (Xian et al. 2004). Replacement of the primary antibody with $1 \%$ BSA in PBS or a normal IgG at the same concentration was used as a negative control. Immunopositive cells within the growth plate injury site were analysed on representative images captured using image analysis software (Image-Pro Plus, Media Cybernetics).

\section{Tibial length measurements of day- 60 rats}

To examine the longer term effects of growth plate injury and anti-VEGF treatment on overall bone longitudinal growth, length measurements before injury and at the time of sample collection were conducted on the right and left tibiae of day 60 animals. The measurements from each group were averaged and expressed as growth (day 60 length - day 0 length) $(\mathrm{cm})$.

\section{Statistical analysis}

Data are presented as mean \pm s.E.M. Comparisons between anti-VEGF- and vehicle-treated groups and between different time points were analysed using two-way ANOVA. When significance levels $(P<0.05)$ were achieved, a post hoc analysis of groups was done using a Tukey's test. In figures, statistical values are presented as ${ }^{*} P<0.05,{ }^{* *} P<0.01,{ }^{* * *} P<0.001$ and ${ }^{* * *} P<0.0001$.

\section{Results}

\section{Serum levels of anti-VEGF delivered systemically}

An ELISA was performed to detect systemic levels of antiVEGF Bevacizumab in serum samples collected on days 6 and 14 following administration. ELISA revealed that antiVEGF remained detectable in both day- 6 and day- 14 serum samples, with the levels being higher on day 14 than those on day 6 (Fig. 1).

Treatment effects on angiogenesis at growth plate injury site and metaphysis

As a means to examine treatment effects on angiogenesis within the injury site, histochemical analysis of the endo-

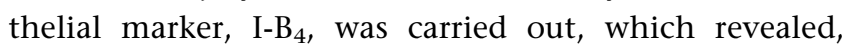

Published by Bioscientifica Ltd. 


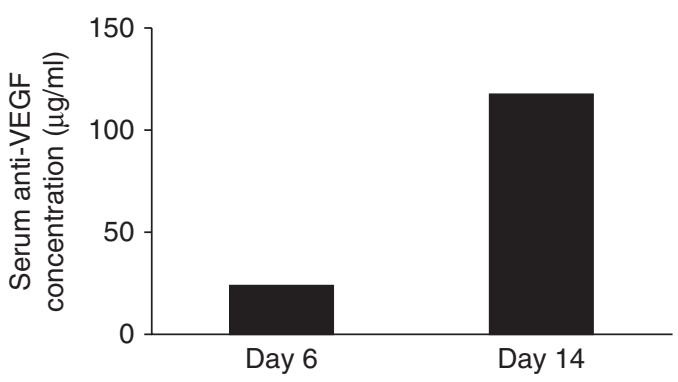

Figure 1

Levels of anti-VEGF in serum of rats at both time points after i.p. injection of the anti-VEGF antibody Bevacizumab at $2.5 \mathrm{mg} / \mathrm{kg}$.

in comparison to injured rats treated with vehicle alone, a visually weaker staining and a decrease in the number of $\mathrm{I}_{-} \mathrm{B}_{4}$-positive cells in the growth plate injury site of anti-VEGF-treated rats on day 6 (Fig. 2A and B). In addition, on day 14 Bevacizumab-treated rats also showed fewer 'vessel-like' structures formed by I-B ${ }_{4}$-positive cells within the growth plate injury site in comparison with control rats that received vehicle (Fig. 2C and D), with the average numbers of vessel-like structures formed being significantly decreased on day 14 following anti-VEGF treatment in comparison with control rats that received vehicle ( $4.84 \mathrm{vs}$ $\left.7.56 / \mathrm{mm}^{2}\right)(P<0.05$; Fig. 2E). Further examination of the metaphyseal regions adjacent to the growth plate injury site also revealed a weaker staining and lower numbers of $\mathrm{I}_{-} \mathrm{B}_{4}$-positive cells and tube-like structures following anti-VEGF treatment in comparison to the injured and vehicle-treated controls (Fig. $2 \mathrm{~F}$ and G). Overall, anti-VEGF treatment seemed to have decreased the population of $\mathrm{I}_{-} \mathrm{B}_{4^{-}}$ positive cells and the blood-vessel-like structures formed at both the growth plate injury site and in the adjacent metaphyseal bone in comparison with those of injured and vehicle-treated control rats, indicating the effectiveness of attenuating angiogenesis by anti-VEGF treatment.

\section{Effects on tissue repair at growth plate injury site}

Basic H\&E and Alcian blue staining of the injury sites of vehicle- and anti-VEGF-treated groups revealed three types of repair tissues: Me, BT and Ca tissues (Fig. 3A, B, C and D). Quantitative histological measurements revealed that treatment with anti-VEGF resulted in significantly greater percentage portions of undifferentiated Me tissue in comparison with the animals that received vehicle at day $14(P<0.01$; 50.7 vs $11.3 \%$; Fig. 3E). Furthermore, treatment with antiVEGF also resulted in a significantly lower percentage of BT repair tissue than in the control animals that received vehicle $(P<0.01 ; 13.0$ vs $50.7 \%)$ on day 14 (Fig. 3E). No statistically significant differences were observed in proportions of $\mathrm{Ca}$ repair tissue at either time point ( $P>0.05$; Fig. 3E).

\section{Effects on bony repair within the growth plate injury site} and drill tract

MicroCT scans revealed bone formation within the growth plate site of vehicle- and anti-VEGF-treated rats at both the day-14 and day-60 time points (Fig. 4A, B, C and D). Consistently, analysis of bone volume at the growth plate injury site showed a significant decrease of $\sim 52 \%$ in bony repair tissue on day 14 growth plate injury sites following anti-VEGF treatment in comparison to the control animals that received vehicle ( 10.4 vs $21.8 \% ; P<0.001)$. This trend was also observed in day 60 rats where the bone volume fraction within the growth plate injury site remained significantly less (with a 19\% decrease) in anti-VEGF-treated rats in comparison with rats that received only vehicle (21.9 vs $27.0 \% ; P<0.01$; Fig. 4E). Furthermore, analysis of the drill tract (from the cortical entry to the metaphysis injury track) also found a significant decrease (34\%) in bone volume present within the injury track in the anti-VEGFtreated rats compared with animals that received only vehicle for day 60 rats (20.8 vs $31.7 \% ; P<0.01$; Fig. 4 F).

\section{Effects on the expression of bone and cartilage-related genes}

Quantitative RT-PCR gene expression analysis of the growth plate injury site revealed a trend towards increased mRNA expression of osteogenesis-related genes - the early transcription factor Runx2 and the late osteogenic marker osteocalcin - following growth plate injury, particularly on day 14 (a day with more active formation). Interestingly, this trend of increased Runx 2 expression appears to be blocked by the anti-VEGF treatment when compared with the vehicle control on day 14 . Treatment with antiVEGF significantly lowered osteocalcin expression back to the 'baseline' non-injured control level in comparison to the vehicle group $(P<0.05$; Fig. $5 \mathrm{~A}$ and $\mathrm{B})$. Consistently, immunolocalisation analysis of RUNX2 within the growth plate injury site showed an observable decrease in the number of RUNX2-immunopositive cells following antiVEGF treatment in comparison with the controls that received only vehicle at both day 6 (Fig. 5C and D) and day 14 time points (Fig. 5E and F).

No significant differences were noted for chondrogenesisrelated genes - early transcription factor Sox 9 and cartilage matrix protein $\operatorname{Col} 2 \mathrm{~A}$, although a slight up-regulation trend was noticed in rats treated with anti-VEGF compared

Published by Bioscientifica Ltd. 

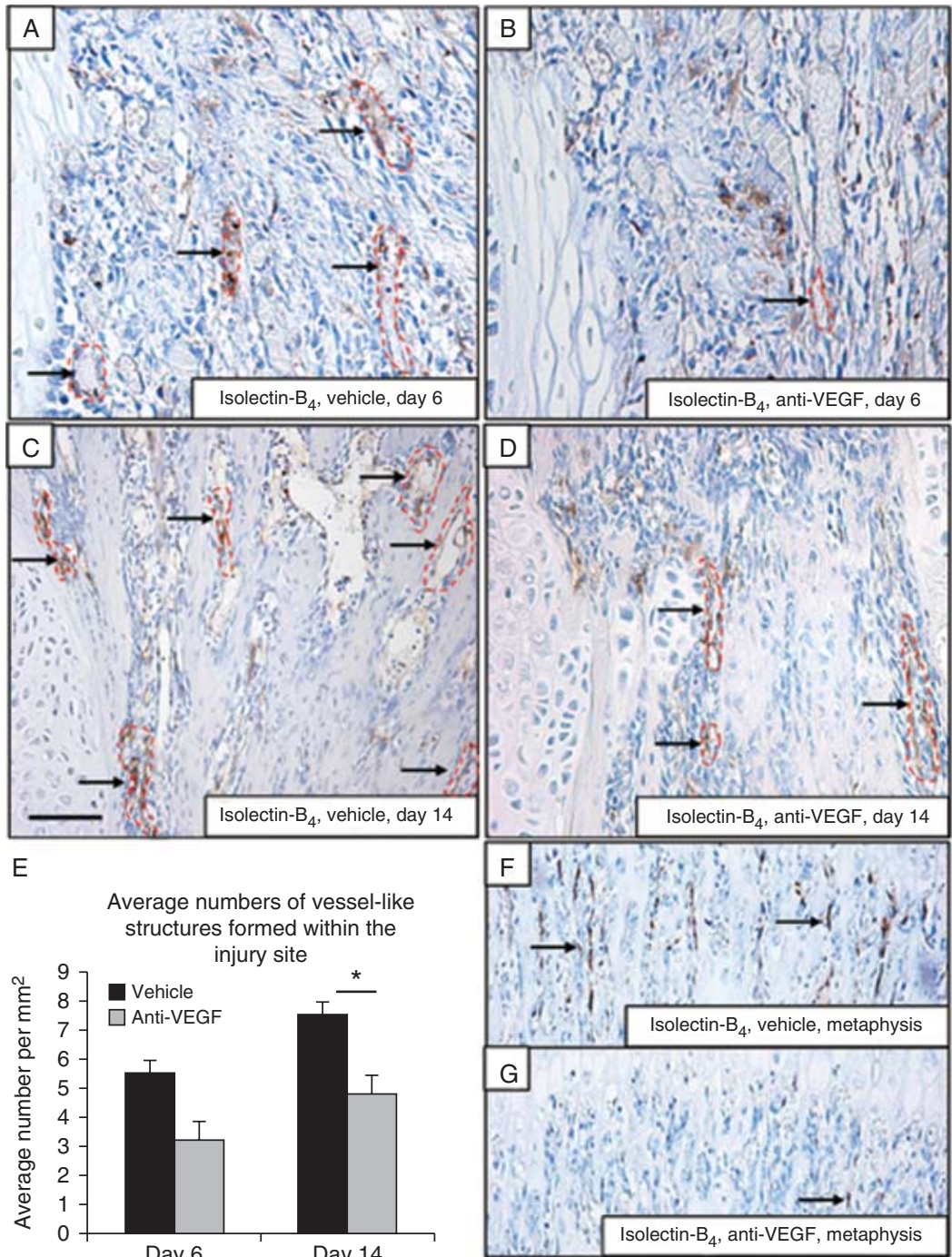

Figure 2

Effects of inhibition of VEGF by anti-VEGF antibody Bevacizumab on angiogenesis within the growth plate injury site and adjacent metaphyseal region as shown by immunostaining of endothelial marker isolectin- $B_{4}$ $\left(\mathrm{I}-\mathrm{B}_{4}\right)$. Representative growth plate injury sites from day 6 vehicle-treated rats $(A)$ and Bevacizumab-treated rats (B), as well as day 14 vehicle-treated rats (C) and Bevacizumab-treated rats (D). I- $\mathrm{B}_{4}$-positive vessel-like structures

with controls that received vehicle. Levels of expression at the injury site (containing repair tissues) of both genes of the injured groups (vehicle- and VEGF-treated) remained below the expression levels for the non-injured controls (with intact growth plate) (Fig. 6A and B).

\section{Effects of injury and treatment on overall tibial bone lengthening}

To examine the overall effects of growth plate injury and anti-VEGF treatment on the growth plate and tibia growth,

\begin{tabular}{|lr} 
http://joe.endocrinology-journals.org & ( 2014 Society for Endocrinology \\
DOI: $10.1530 / J O E-13-0539$ & Printed in Great Britain
\end{tabular}

are indicated with red dotted lines and arrows (A, B, C and D) and average numbers within the growth plate injury site were quantified $(E)$ ( ${ }^{*} P<0.05$, Bevacizumab-treated group vs vehicle-treated groups). Representative metaphyseal regions from day 6 vehicle-treated rats $(F)$ and Bevacizumab-treated rats (G). Original scale bars $=125 \mu \mathrm{m}$ ( $A$ and $B$ ) and $250 \mu \mathrm{m}$ (C, D, F and G).

length measurements were taken on both tibiae of day 60 group rats before surgery and on the day of sample collection. No obvious differences were observed between the left and right tibiae in the uninjured groups of either untreated (Fig. 7A) or anti-VEGF treated (Fig. 7B) rats; for quantitative analysis, measurements of growth from the left and right tibiae were collated and averaged for further comparison with other treatment groups. However, some length differences were observed between the injured right tibiae and the uninjured left tibiae of both the vehicle-treated group (Fig. 7C) and the anti-VEGF-treated 

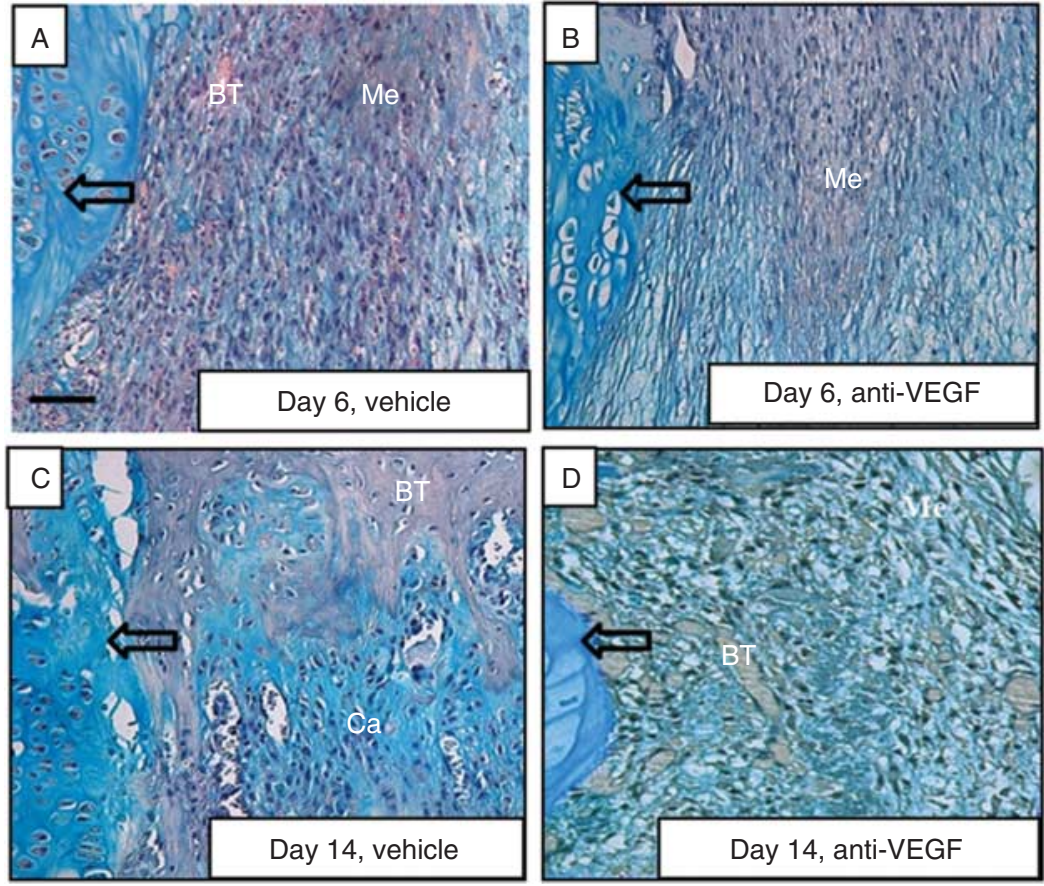

E

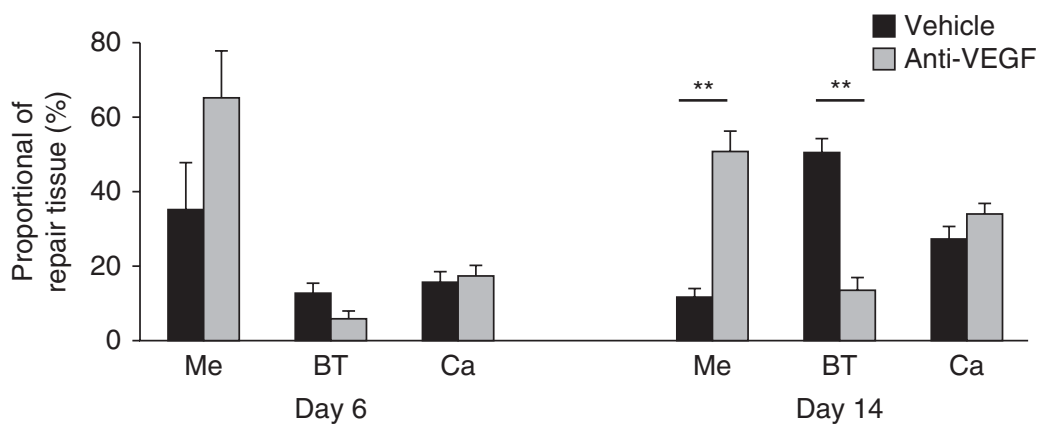

Figure 3

Effects of anti-VEGF antibody Bevacizumab treatment on proportions of repair tissues within the growth plate injury site. H\&E and Alcian bluestained injury sites from day 6 and day 14 vehicle-treated rats (A and $C$ ) and anti-VEGF-treated rats ( $B$ and $D)$, showing mesenchymal (Me) tissue, bony trabeculae (BT) and cartilaginous (Ca) tissue as distinguished by cell colour and morphology. The remaining adjacent growth plate cartilage is

group (Fig. 7D). More specifically, in comparison to the untreated and uninjured group, there was a significant decrease in the averaged tibial growth in the uninjured and anti-VEGF-treated rats ( $2.61 \mathrm{vs} 2.04 \mathrm{~cm} ; P<0.05)$. Furthermore, in comparison to the uninjured and untreated group, significant decreases in tibial length were also observed in the right injured tibiae of vehicle-treated rats $(2.6 \mathrm{vs} 1.98 \mathrm{~cm}$; $P<0.001$ ) as well as both the uninjured left and injured right tibiae from the anti-VEGF-treated rats $(2.6 \mathrm{vs} 1.68 \mathrm{~cm}$ and 2.6 vs $1.25 \mathrm{~cm}$ respectively; $P<0.001)$. In addition, compared with the averaged tibial growth of the uninjured indicated by open arrows. Original scale bar $=250 \mu \mathrm{m}$. Quantitative histological image analysis measurements of area percentage (of the total growth plate injury site areas, $n=8$ ) showing a significant increase in Me tissue at day 14 post injury $(* * P<0.01)$, a significant decrease in bony trabecular repair tissue at day $14(* * P<0.01)$ and no differences in Ca repair tissue proportions for both time-points and treatments $(P>0.05)(E)$.

and anti-VEGF-treated groups, there was a significant decrease in the right injured tibiae of anti-VEGF-treated rats ( 2.04 vs $1.25 \mathrm{~cm} ; P<0.001)$. Interestingly, a significant decrease was also observed in the growth of uninjured left tibiae of anti-VEGF-treated rats in comparison with the left uninjured tibiae of vehicle-treated rats $(1.68$ vs $2.23 \mathrm{~cm}$; $P<0.01)$. A similar significant decrease was also seen in the growth of right tibiae of these groups $(1.25 \mathrm{vs} 1.98 \mathrm{~cm}$; $P<0.001)$. Lastly, a significant decrease in tibial growth was found between the uninjured left tibiae and the injured right tibiae of the anti-VEGF group ( $1.68 \mathrm{vs} 1.25 \mathrm{~cm} ; P<0.05)$. 

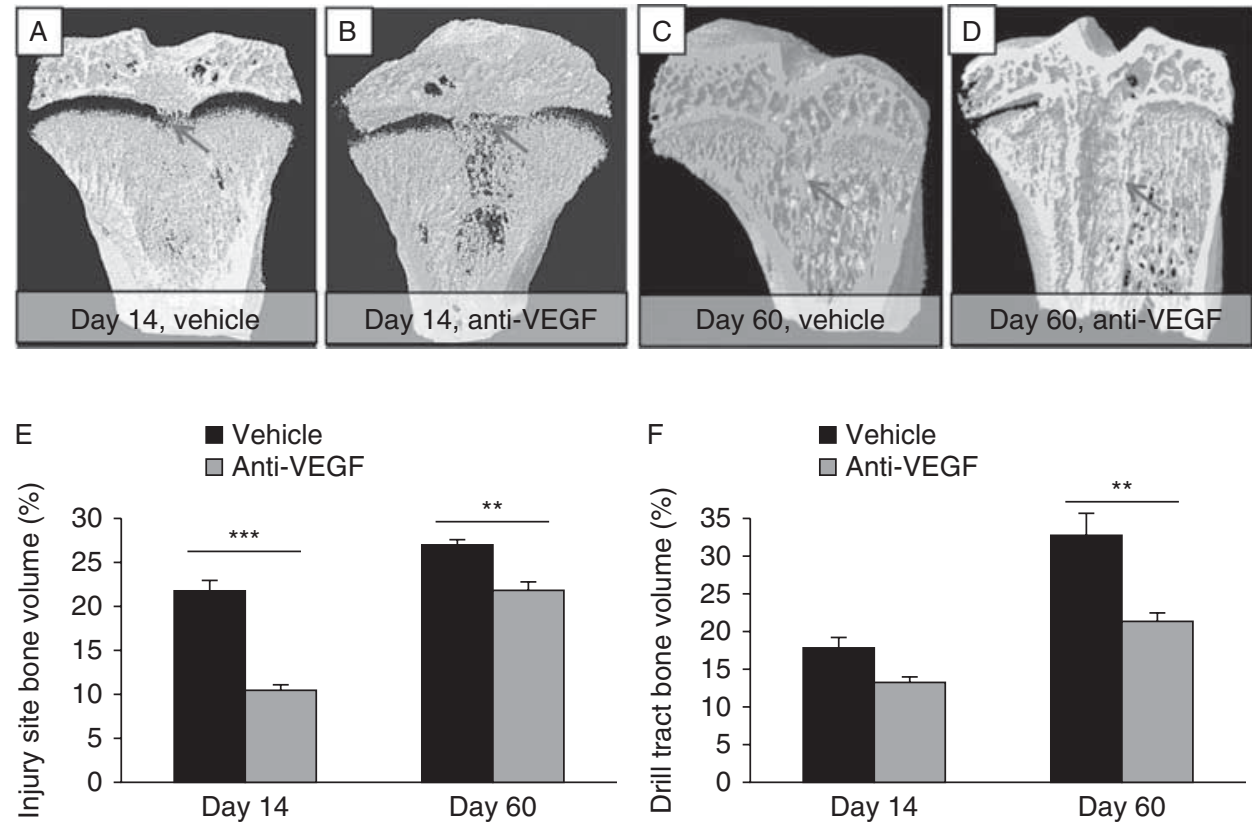

Figure 4

MicroCT analysis of treatment effects with anti-VEGF antibody Bevacizumab on bone volume fraction within growth plate injury site and drill tract at days 14 and 60 post injury. Growth plate microCT images from a day 14 injured vehicle-treated rat (A), day 14 injured anti-VEGF-treated rat (B), a day 60 injured vehicle-treated rat (C) and a day 60 injured antiVEGF-treated rat (D); calculation of the bone volume over tissue volume

(BV/TV\%) within the injury site (grey arrows) at days 14 and 60 (E), showing a significant reduction in the anti-VEGF-treated groups compared with the vehicle control ( $* * * P<0.001$ and $* * P<0.01)$. MicroCT analysis of the drill tract directly under the growth plate injury site (grey arrow) showed a significant decrease in BV/TV\% following treatment with the anti-VEGF in day 60 post-injury rats $(* * P<0.01)(\mathrm{F})$.

\section{Treatment effects on the zonal heights of the adjacent growth plate cartilage}

As a means to examine the effects of anti-VEGF treatment on growth plate, height measurements of growth plate zones revealed that, in comparison with the uninjured controls (Fig. 8A) and the injured rats treated with the vehicle control (Fig. 8B), anti-VEGF treatment (Fig. 8C) caused a significant increase in the height of the hypertrophic zone $(P<0.05)(0.24,0.25$ and $1.27 \mathrm{~mm}$, respectively, for uninjured control, vehicle-treated and anti-VEGF-treated groups) (Fig. 8D and E). No significant zonal height differences were found between the uninjured control and vehicle-treated injured groups $(P>0.05)$. However, the anti-VEGF treatment did not cause alterations in heights of the resting and proliferative zones of any of the groups $(P>0.05$; Fig. $8 \mathrm{E})$.

\section{Discussion}

Currently, the underlying pathophysiology for the bony repair of the injured growth plate cartilage remains unclear. Since VEGF is critical for angiogenesis during bone fracture

repair (Ogden 2000a,b,c, Keramaris et al. 2008, Beamer et al. 2010) and angiogenesis of the growth plate injury site is a prerequisite for the fibrous repair tissue to convert to bony repair (Ogden 2000a,b,c), we proposed the hypothesis that VEGF plays a role in the undesirable bone repair of the injured growth plate. Using a rat tibial growth plate injury model together with a clinically used anti-VEGF neutralising antibody, Bevazicumab, the current study showed that VEGF is important for bone formation at the growth plate injury site as blockage of VEGF using the neutralising antibody results in a significant decrease in bony repair tissue formed but a significant increase in the remaining Me repair tissue at the growth plate injury site.

Suggestive of successful delivery of anti-VEGF to the circulation, serum collected from rats treated on both days 6 and 14 showed detectable levels of anti-VEGF. Unexpectedly, the amount of anti-VEGF detected at day 14 was greater than that at day 6 , although it is unknown whether the serum concentrations are correlated with the bioactivity at the two time points. This higher level of anti-VEGF could be due to factors including the pharmacokinetics of the anti-VEGF delivered. Interestingly, other

Published by Bioscientifica Ltd. 

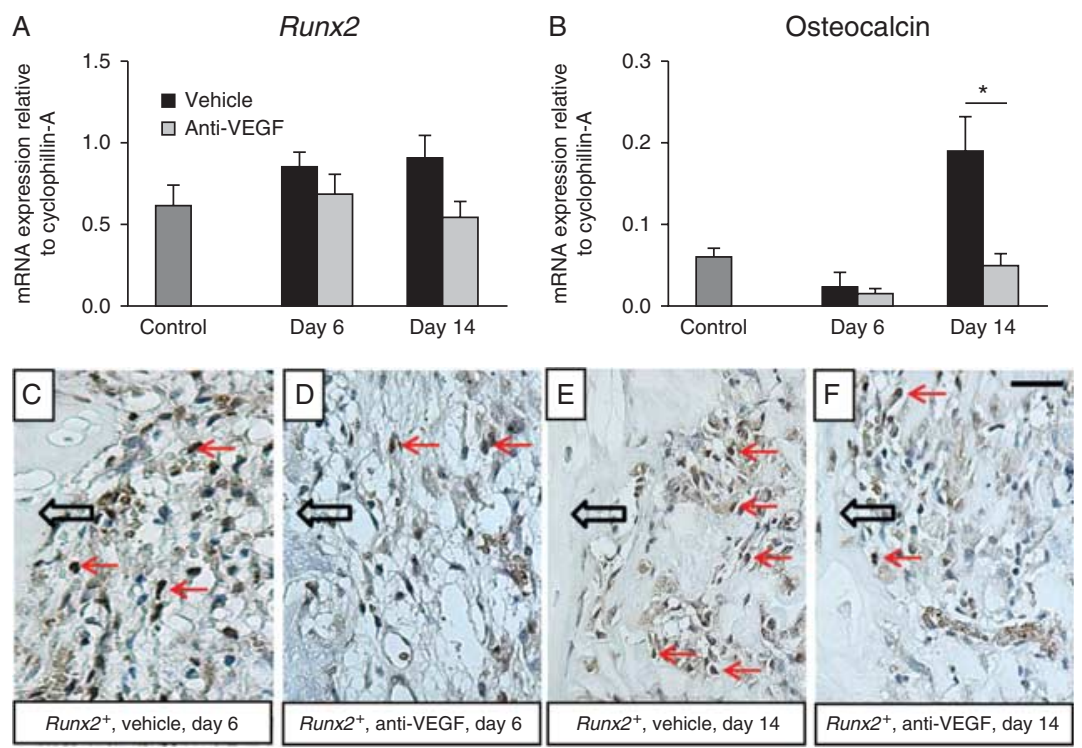

\section{Figure 5}

Effects of anti-VEGF antibody Bevacizumab treatment on mRNA expression of genes involved with osteogenic repair at the injured growth plate. Quantitative real-time RT-PCR expression data for Runx2 (A) and osteocalcin (B) are expressed relative to internal standard Cyclophillin-A ( ${ }^{*} P<0.05$, Bevacizumab-treated group vs vehicle group).

studies conducted in rabbits and human infants have also detected serum levels of anti-VEGF in a similar pattern, with peak systemic levels detected between days 7 and 14 post administration (Wu et al. 2010, Sato et al. 2012). Overall, due to its extended half-life and specificity for blocking VEGF from binding to its receptor, Bevazicumab was an ideal tool for this current study to establish a potential role for VEGF in the undesirable bony repair following growth plate injuries.

Indicative of its bioactivity in inhibiting VEGF and the role of VEGF in angiogenesis of the growth plate injury site, the current study observed a significant reduction in I- $\mathrm{B}_{4}$-labelled blood vessel-like structures within the growth plate injury site and the surrounding area of treated rats. VEGF has an important role in the migration and proliferation of endothelial cells (Carlevaro et al. 2000); hence, it is not surprising that its neutralisation caused a noticeable decrease in numbers of $\mathrm{I}_{-} \mathrm{B}_{4}$-positive endothelial cells observed within the growth plate injury site on days 6 and 14 with a significant decrease in the number of 'blood vessel-like' structures formed by $\mathrm{I}_{-} \mathrm{B}_{4}$-positive cells in the anti-VEGF-treated rats. Apart from the growth plate injury site, anti-VEGF treatment was also shown to decrease the number of $\mathrm{I}_{-} \mathrm{B}_{4}$ cells in the metaphyseal region. In addition, in the uninjured growth plate region adjacent to the growth plate injury site itself, there was
RUNX2-immunopositive cells were detected within the growth plate injury site (indicated by red arrows) of both vehicle- and anti-VEGFtreated rats at days 6 and 14 (C, D, E and F). The remaining adjacent growth plate cartilage is indicated on the figure by open arrows. Original scale bar $=125 \mu \mathrm{m}$ (applies to C, D, E and F).
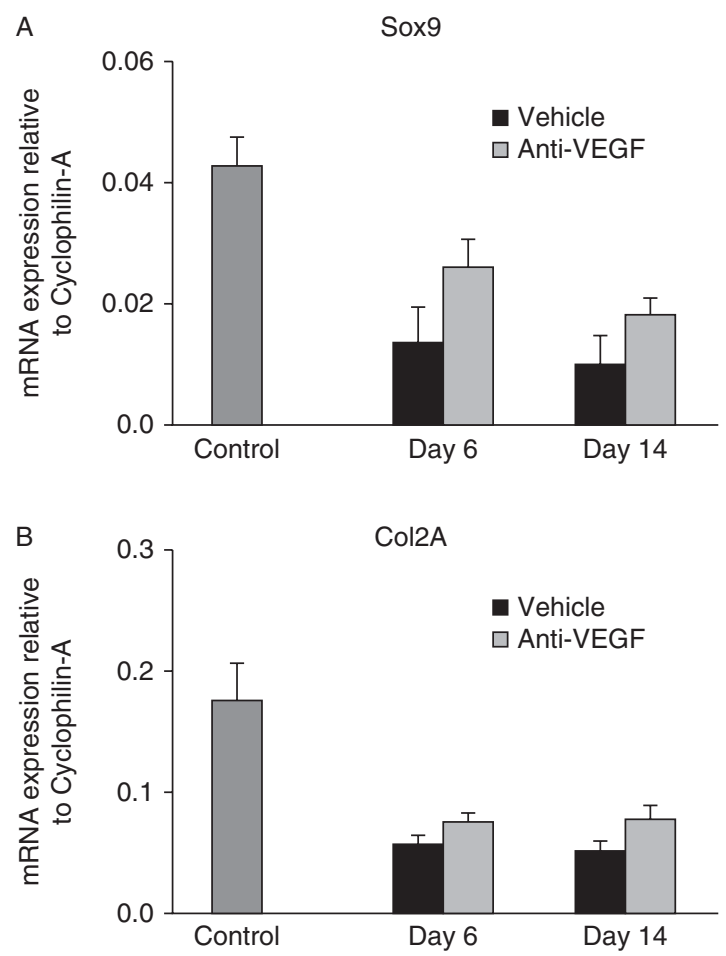

Figure 6

Effects of anti-VEGF antibody Bevacizumab treatment on mRNA expression of genes involved in cartilaginous repair at the injured growth plate. Quantitative real-time RT-PCR expression data for Sox9 (A) and Col2A (B) are expressed relative to internal standard Cyclophilin-A. 


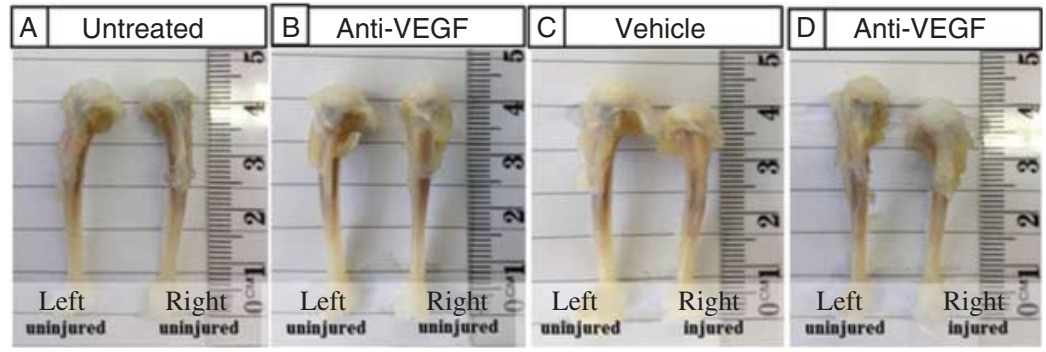

$E$

Tibial growth post-surgery (60 days)
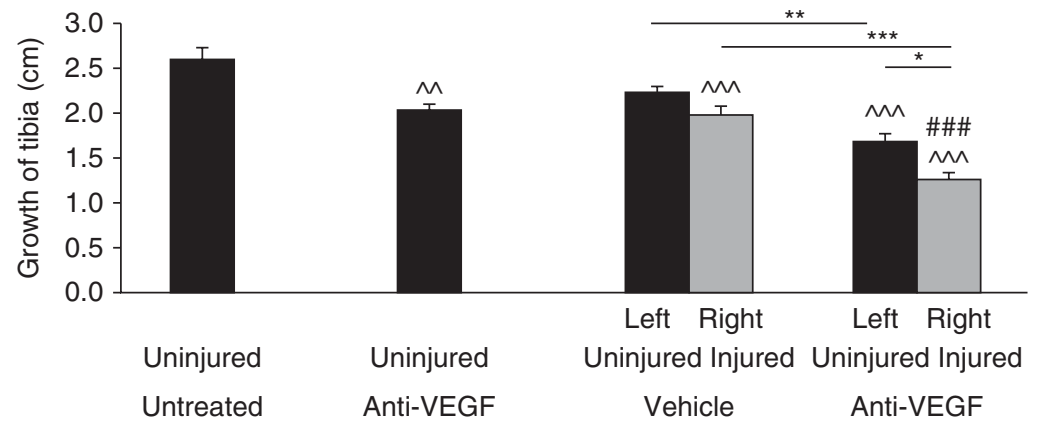

Untreated

Anti-VEGF

Uninjured Injured Uninjured Injured

Vehicle

Anti-VEGF

\section{Figure 7}

Effects of anti-VEGF antibody Bevacizumab treatment on overall tibial growth in day 60 post-surgery rats. Representative images show the right and left tibiae of untreated (A), anti-VEGF-treated (B), uninjured left and injured right tibia of injured vehicle-treated $(C)$ and uninjured left and injured right tibia of injured anti-VEGF-treated rats (D). Analysis of tibial growth post surgery, showing, in comparison to the untreated rats,

a visual decrease in vascular invasion of the adjacent growth plate and thus an expansion of the hypertrophic zone of the growth plate. This is consistent with previous observations that VEGF is mainly produced by hypertrophic chondrocytes (Gerber et al. 1999) and its inhibition can significantly impair vascular invasion of the growth plate hypertrophic zone (Haigh et al. 2000). However, in the current study, although there was an overall reduction in $\mathrm{I}_{-} \mathrm{B}_{4}$-positive cells and -labelled blood vessel-like structures, they could still be found at the growth plate injury site in treated rats. It is possible that the dose or method of delivery might be inadequate to completely halt angiogenesis within the injury site although a considerable level of anti-VEGF in the circulation could be detected at the two time points analysed. In addition, it could also be possible that VEGF may not be the only key regulator driving angiogenesis and there may be other factors and mechanisms which drive blood vessel formation at the injured growth plate. Furthermore, apart from the current histology-based method of detecting $\mathrm{I}_{-} \mathrm{B}_{4}$-positive cells and -labelled blood vessel-like structures, additional quantitative methods may be useful in future studies for analysing significant decreases in growth following anti-VEGF treatment alone as well as vehicle and anti-VEGF treatments with growth plate injuries $(E)$ $\left({ }^{*}, * *\right.$, or ${ }^{* * *}$ comparison to uninjured and untreated group ${ }^{\wedge \wedge}$ or ${ }^{\wedge \wedge}$ comparison to the untreated control; and \#\#\# comparison to uninjured anti-VEGF-treated group) $\left({ }^{*} P<0.05,{ }^{\wedge}, * * P<0.01\right.$ and

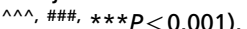

the treatment effects on angiogenesis. Nonetheless, taken together, our data indicate effectiveness of this neutralising antibody in inhibiting and the role of VEGF in promoting angiogenesis at the growth plate injury site. Similarly, the inhibition of VEGF was also previously observed to decrease blood flow and to halt fracture repair in a rabbit radial fracture model (Wang et al. 2010).

Consistent with the critical role of VEGF in bone healing in bone fracture models, as assessed by microCT analysis, neutralisation of VEGF resulted in a significant decrease in the volume of bony repair tissue at the growth plate injury site on days 14 and 60 post injury. This was further supported by quantitative histological image analysis in which a significant decrease in the percentage of bony trabecular repair tissue was found in treated rats when compared with the control group that received vehicle. Consistently, microCT analysis of bone volume within the injury drill tract also showed signs of reduced bony repair in the anti-VEGF-treated group. Interestingly, this significant decrease in bony repair was accompanied by an observed significant increase in the amount of undifferentiated Me tissue present within the growth plate injury site. Previous studies have shown that during bone

Published by Bioscientifica Ltd. 

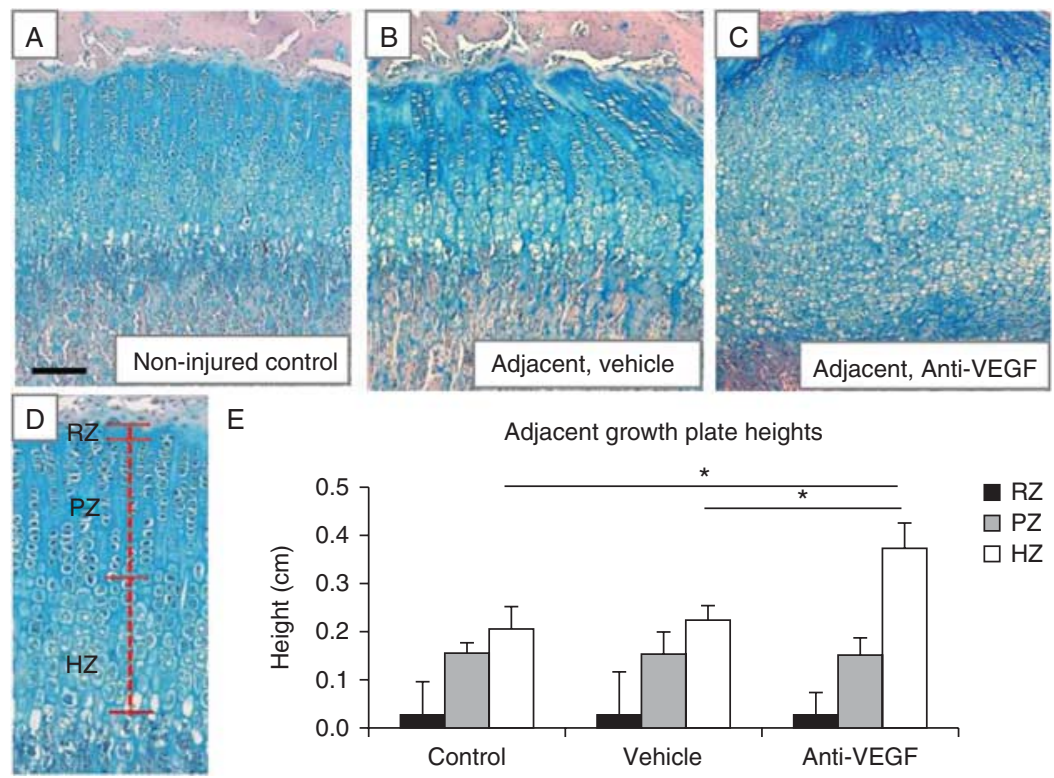

\section{Figure 8}

Hypertrophic zone expansion at the regions of growth plate adjacent to the growth plate injury site. In comparison to uninjured controls (A) and injured vehicle-treated controls (B), the systemic delivery of anti-VEGF agent Bevacizumab caused an obvious increase in the hypertrophic zone of the regions of growth plate adjacent to the growth plate injury site (C). Following measurements of heights of the resting zone (RZ), proliferative zone (PZ) and hypertrophic zone (HZ) (D), image analysis quantification observed a significant increase in the hypertrophic zonal height of anti-VEGF-treated group in comparison to uninjured controls and injured controls that received vehicle (E) $\left({ }^{*} P<0.05\right)$. Original scale bar $=125 \mu \mathrm{m}$ (applies to $A, B$ and $C$ ). fracture healing, some infiltrated Me cells retain the ability to undergo chondrogenic and/or osteogenic differentiation (Aubin 1999, Solchaga et al. 1999), and that, following anti-angiogenic treatment in a rat fracture model, there was a severely impaired fracture healing response and an absence of cartilage differentiation (Hausman et al. 2001). These data indicate that, similar to bone fracture healing, VEGF is critical for the conversion of Me tissue to bony tissue and its inhibition will result in suppressed or delayed bony repair in both bone fracture healing and growth plate repair.

Consistent with the reduced bony repair outcome in anti-VEGF-treated rats, there was a significant decrease in mRNA expression of the late osteogenic marker, osteocalcin, as well as an observable decreasing trend for the earlier osteogenic transcription factor, Runx2, following antiVEGF treatment when compared with their vehicle-treated counterparts. This trend was further supported through immunohistochemical data which showed a decrease in the number of RUNX2-immunopositive cells at the growth plate injury site following anti-VEGF treatment on both days 6 and 14 post injury. Similarly, a more recent study by Liu et al. (2012) has shown a significant decrease in RUNX2 protein expression in cells which expressed VEGF-specific short hairpin RNA (Liu et al. 2012), elucidating the association between decreased VEGF and low levels of RUNX2 protein as a possible explanation for the reduction in osteoblast differentiation (Liu et al. 2012). Conversely, despite lacking statistical significance, a trend towards increased expression was noted at both time points for early and late chondrogenesis-related genes, Sox 9 and Col2A respectively. Further research is required to investigate whether inhibiting angiogenesis and bone formation could lead to an increased amount of cartilage repair tissue being present.

However, since a high VEGF level during the late stage of chondrogenesis is known to promote endochondral ossification to convert hypertrophic cartilage to become bone (Carlevaro et al. 2000), it is possible that inhibition of VEGF angiogenesis could cause suppressed conversion of cartilage into bone at the growth plate injury site. In support, and indicative of VEGF's importance during endochondral ossification and bone lengthening, examination of the day 60 rats showed that all groups that received growth plate injury had significantly shorter limbs when compared with those without injury. Moreover, when combined with anti-VEGF treatment, growth plate injury further reduced tibial bone lengthening. Consistent with this possibility, systemic blocking of VEGF was found in the current study to also result in a

Published by Bioscientifica Ltd. 
significant expansion of the hypertrophic zone of the intact growth plate cartilage adjacent to the growth plate injury site. Similarly, it was shown previously that the absence of VEGF in growing mice resulted in a greater hypertrophic zone of the growth plate and poorer trabecular bone formation in the metaphysis (Gerber et al. 1999, Ryan et al. 1999).

In summary, data from the current and previous studies indicate that VEGF-induced angiogenesis is important not only in bone formation at the growth plate injury site but also in the endochondral ossification and conversion of hypertrophic cartilage to trabecular bone. However, future studies involving a more local and direct method of VEGF inhibition at the growth plate injury site may further clarify the role of VEGF-induced angiogenesis for the undesirable bony repair tissue formation. Future studies should be conducted to investigate whether this VEGF neutralising antibody (Bevazicumab) can be delivered locally to the growth plate injury site to block the unwanted 'faulty' bony repair and to limit potential systemic adverse effects of this drug. In addition, before such an agent can be used therapeutically, more thorough studies will be required to examine the potential side effects and concerns occurring from its use.

\section{Declaration of interest}

The authors declare that there is no conflict of interest that could be perceived as prejudicing the impartiality of the research reported.

\section{Funding}

This project was funded in parts by grants from the Bone Health foundation, Channel-7 Children's Research Foundation of South Australia, a University of South Australia Division of Health Science Research Development Grant, and the Australian National Health and Medical Research Council (NHMRC). C J X is a Senior Research Fellow of NHMRC Australia.

\section{References}

Arasapam G, Scherer M, Cool JC, Foster BK \& Xian CJ 2006 Roles of COX-2 and iNOS in the bony repair of the injured growth plate cartilage. Journal of Cellular Biochemistry 99 450-461. (doi:10.1002/jcb.20905)

Aubin JE 1999 Osteoprogenitor cell frequency in rat bone marrow stromal populations: role for heterotypic cell-cell interactions in osteoblast differentiation. Journal of Cellular Biochemistry 72 396-410. (doi:10.1002/ (SICI)1097-4644(19990301)72:3\%3C;396::AID-JCB9\%3E;3.0.CO;2-6)

Beamer B, Hettrich C \& Lane J 2010 Vascular endothelial growth factor: an essential component of angiogenesis and fracture healing. HSS Journal 6 85-94. (doi:10.1007/s11420-009-9129-4)

Carano RA \& Filvaroff EH 2003 Angiogenesis and bone repair. Drug Discovery Today 8 980-989. (doi:10.1016/S1359-6446(03)02866-6)

Carlevaro MF, Cermelli S, Cancedda R \& Descalzi Cancedda F 2000 Vascular endothelial growth factor (VEGF) in cartilage neovascularization and

http://joe.endocrinology-journals.org DOI: 10.1530/JOE-13-0539
() 2014 Society for Endocrinology Printed in Great Britain chondrocyte differentiation: auto-paracrine role during endochondral bone formation. Journal of Cell Science 113 59-69.

Chung R, Cool JC, Scherer MA, Foster BK \& Xian CJ 2006 Roles of neutrophil-mediated inflammatory response in the bony repair of injured growth plate cartilage in young rats. Journal of Leukocyte Biology 80 1272-1280. (doi:10.1189/jlb.0606365)

Chung R, Foster BK, Zannettino AC \& Xian CJ 2009 Potential roles of growth factor PDGF-BB in the bony repair of injured growth plate. Bone 44 878-885. (doi:10.1016/j.bone.2009.01.377)

Chung R, Foster BK \& Xian CJ 2011 Preclinical studies on mesenchymal stem cell-based therapy for growth plate cartilage injury repair. Stem Cells International 2011570125 . (doi:10.4061/2011/570125)

Chung R, Foster BK \& Xian CJ $2013 a$ Inhibition of protein kinase-D promotes cartilage repair at injured growth plate in rats. Injury $\mathbf{4 4}$ 914-922. (doi:10.1016/j.injury.2013.01.038)

Chung R, Wong D, Macsai C, Piergentili A, Del Bello F, Quaglia W \& Xian CJ $2013 b$ Roles of Wnt/ $\beta$-catenin signalling pathway in the bony repair of injured growth plate cartilage in young rats. Bone 52 651-658. (doi:10.1016/j.bone.2012.10.035)

Deckers MM, Karperien M, van der Bent C, Yamashita T, Papapoulos SE \& Lowik CW 2000 Expression of vascular endothelial growth factors and their receptors during osteoblast differentiation. Endocrinology 141 1667-1674. (doi:10.1210/endo.141.5.7458)

Fan C, Cool JC, Scherer MA, Foster BK, Shandala T, Tapp H \& Xian CJ 2009 Damaging effects of chronic low-dose methotrexate usage on primary bone formation in young rats and potential protective effects of folinic acid supplementary treatment. Bone $\mathbf{4 4}$ 61-70. (doi:10.1016/j.bone. 2008.09.014)

Ferguson C, Alpern E, Miclau T \& Helms JA 1999 Does adult fracture repair recapitulate embryonic skeletal formation? Mechanisms of Development 87 57-66. (doi:10.1016/S0925-4773(99)00142-2)

Fischerauer E, Heidari N, Neumayer B, Deutsch A \& Weinberg AM 2011 The spatial and temporal expression of VEGF and its receptors 1 and 2 in post-traumatic bone bridge formation of the growth plate. Journal of Molecular Histology 42 513-522. (doi:10.1007/s10735-011-9359-x)

Geiger F, Bertram H, Berger I, Lorenz H, Wall O, Eckhardt C, Simank HG \& Richter W 2005 Vascular endothelial growth factor gene-activated matrix (VEGF165-GAM) enhances osteogenesis and angiogenesis in large segmental bone defects. Journal of Bone and Mineral Research 20 2028-2035. (doi:10.1359/JBMR.050701)

Gerber HP, Vu TH, Ryan AM, Kowalski J, Werb Z \& Ferrara N 1999 VEGF couples hypertrophic cartilage remodeling, ossification and angiogenesis during endochondral bone formation. Nature Medicine $\mathbf{5}$ 623-628. (doi:10.1038/9467)

Haigh JJ, Gerber HP, Ferrara N \& Wagner EF 2000 Conditional inactivation of VEGF-A in areas of collagen2a1 expression results in embryonic lethality in the heterozygous state. Development 127 1445-1453.

Hausman MR, Schaffler MB \& Majeska RJ 2001 Prevention of fracture healing in rats by an inhibitor of angiogenesis. Bone 29 560-564. (doi:10.1016/S8756-3282(01)00608-1)

Ignjatovic D, Aasland K, Pettersen M, Sund S, Chen Y, Spasojevic M \& Nesgaard JM 2010 Intra-abdominal administration of bevacizumab diminishes intra-peritoneal adhesions. American Journal of Surgery 200 270-275. (doi:10.1016/j.amjsurg.2009.08.038)

Keramaris N, Calori G, Nikolaou V, Schemitsch E \& Giannoudis P 2008 Fracture vascularity and bone healing: a systematic review of the role of VEGF. Injury 39 S45-S57. (doi:10.1016/S0020-1383(08)70015-9)

Lin YS, Nguyen C, Mendoza JL, Escandon E, Fei D, Meng YG \& Modi NB 1999 Preclinical pharmacokinetics, interspecies scaling, and tissue distribution of a humanized monoclonal antibody against vascular endothelial growth factor. Journal of Phamacology and Experimental Therapeutics 288 371-378.

Liu Y, Berendsen AD, Jia S, Lotinun S, Baron R, Ferrara N \& Olsen BR 2012 Intracellular VEGF regulates the balance between osteoblast and adipocyte differentiation. Journal of Clinical Investigation 122 3101-3113. (doi:10.1172/JCI61209) 
Macsai CE, Hopwood B, Chung R, Foster BK \& Xian CJ 2011 Structural and molecular analyses of bone bridge formation within the growth plate injury site and cartilage degeneration at the adjacent uninjured area. Bone 49 904-912. (doi:10.1016/j.bone.2011.07.024)

Macsai CE, Georgiou KR, Foster BK, Zannettino AC \& Xian CJ 2012 Microarray expression analysis of genes and pathways involved in growth plate cartilage injury responses and bony repair. Bone $\mathbf{5 0}$ 1081-1091. (doi:10.1016/j.bone.2012.02.013)

McCarty RC, Xian CJ, Gronthos S, Zannettino AC \& Foster BK 2010 Application of autologous bone marrow derived mesenchymal stem cells to an ovine model of growth plate cartilage injury. Open Orthopaedics Journal 4 204-210. (doi:10.2174/1874325001004010204)

Midy V \& Plouet J 1994 Vasculotropin/vascular endothelial growth factor induces differentiation in cultured osteoblasts. Biochemical and Biophysical Research Communications 199 380-386. (doi:10.1006/bbrc.1994.1240)

Mizuta T, Benson WM, Foster BK, Paterson DC \& Morris LL 1987 Statistical analysis of the incidence of physeal injuries. Journal of Pediatric Orthopedics 7 518-523. (doi:10.1097/01241398-198709000-00003)

Nakagawa M, Kaneda T, Arakawa T, Morita S, Sato T, Yomada T, Hanada K, Kumegawa M \& Hakeda Y 2000 Vascular endothelial growth factor (VEGF) directly enhances osteoclastic bone resorption and survival of mature osteoclasts. FEBS Letters 473 161-164. (doi:10.1016/ S0014-5793(00)01520-9)

Ogden JA 2000a Injury to the growth mechanisms. In Skeletal Injury in the Child. third edition, ch 6, pp 147-208 New York: Springer-Verlag.

Ogden JA $2000 b$ Management of growth mechanism injuries and arrest. In Skeletal Injury in the Child. third edition, ch 7, pp 209-242 New York: Springer-Verlag.

Ogden JA 2000c Biology of repair of the immature skeleton. In Skeletal Injury in the Child. third edition, ch 8, pp 243-268 New York: Springer-Verlag.

Ryan AM, Eppler DB, Hagler KE, Bruner RH, Thomford PJ, Hall RL, Shopp GM \& O'Neil CA 1999 Preclinical safety evaluation of rhuMAbVEGF, an antiangiogenic humanized monoclonal antibody. Toxicologic Pathology 27 78-86. (doi:10.1177/019262339902700115)

Salter RB \& Harris WR 1963 Injuries involving the epiphyseal plate. Journal of Bone and Joint Surgery 45-A 587-622.

Sato T, Hanada M, Ohtani M, Nakai T, Teramura M, Sadoya H, Takahashi T \& Hongo A 2012 Short communication: Effect of difructose anhydride III on serum immunoglobulin G concentration in newborn calves. Journal of Dairy Science 95 5336-5339. (doi:10.3168/jds.2011-4989)
Solchaga LA, Dennis JE, Goldberg VM \& Caplan AI 1999 Hyaluronic acidbased polymers as cell carriers for tissue-engineered repair of bone and cartilage. Journal of Orthopaedic Research 17 205-213. (doi:10.1002/ jor.1100170209)

Street J, Bao M, deGuzman L, Bunting S, Peale FV Jr, Ferrara N, Steinmetz H, Hoeffel J, Cleland JL, Daugherty A et al. 2002 Vascular endothelial growth factor stimulates bone repair by promoting angiogenesis and bone turnover. PNAS 99 9656-9661. (doi:10.1073/pnas.152324099)

Tang W, Yang F, Li Y, de Crombrugghe B, Jiao H, Xiao G \& Zhang C 2012 Transcriptional regulation of vascular endothelial growth factor (VEGF) by osteoblast-specific transcription factor Osterix (Osx) in osteoblasts. Journal of Biological Chemistry 287 1671-1678. (doi:10.1074/jbc. M111.288472)

Wang CJ, Huang KE, Sun YC, Yang YJ, Ko JY, Weng LH \& Wang FS 2010 VEGF modulates angiogenesis and osteogenesis in shockwavepromoted fracture healing in rabbits. Journal of Surgical Research $\mathbf{1 7 1}$ 114-119. (doi:10.1016/j.jss.2010.01.045)

Wu WC, Lai CC, Chen KJ, Chen TL, Wang NK, Hwang YS, Yeung L \& Li LM 2010 Long-term tolerability and serum concentration of bevacizumab (avastin) when injected in newborn rabbit eyes. Investigative Ophthalmology \& Visual Science 51 3701-3708. (doi:10.1167/iovs.09-4425)

Xian CJ \& Foster BK 2006 Repair of injured articular and growth plate cartilage using mesenchymal stem cells and chondrogenic gene therapy. Current Stem Cell Research \& Therapy 1 213-229. (doi:10.2174/ $157488806776956904)$

Xian CJ, Zhou FH, McCarty RC \& Foster BK 2004 Intramembranous ossification mechanism for bone bridge formation at the growth plate cartilage injury site. Journal of Orthopaedic Research 22 417-426. (doi:10.1016/j.orthres.2003.08.003)

Zelzer E, Mamluk R, Ferrara N, Johnson RS, Schipani E \& Olsen BR 2004 VEGFA is necessary for chondrocyte survival during bone development. Development 131 2161-2171. (doi:10.1242/dev.01053)

Zhou FH, Foster BK, Sander G \& Xian CJ 2004 Expression of proinflammatory cytokines and growth factors at the injured growth plate cartilage in young rats. Bone 35 1307-1315. (doi:10.1016/ j.bone.2004.09.014)

Zhou FH, Foster BK, Zhou XF, Cowin AJ \& Xian CJ 2006 TNF- $\alpha$ mediates p38 MAP kinase activation and negatively regulates bone formation at the injured growth plate in rats. Journal of Bone and Mineral Research $\mathbf{2 1}$ 1075-1088. (doi:10.1359/jbmr.060410)

Received in final form 7 January 2014

Accepted 23 January 2014

Accepted Preprint published online 24 January 2014
(C) 2014 Society for Endocrinology Printed in Great Britain
Published by Bioscientifica Ltd. 